\section{UP-TITRATION OF SECONDARY PREVENTION FOLLOWING ACUTE CORONARY SYNDROME (ACS)}

${ }^{1}$ Sadeer Fhadil*, ${ }^{1}$ Adam Timmis, ${ }^{2}$ Niket Ruparelia, ${ }^{1}$ Paul Wright, ${ }^{1}$ Riyaz Patel, ${ }^{3}$ Preeti Sud, ${ }^{4} J o h n$ Robson, 'Sotiris Antoniou. ${ }^{1}$ Barts Health NHS Trust; ${ }^{2}$ Purdue University; ${ }^{3} \mathrm{UCL}$ Partners; ${ }^{4}$ Centre for Primary Care and Public Health, Blizard Institute, Queen Mary University of London

\subsection{6/heartjnl-2017-311726.70}

Introduction Optimum doses of secondary prevention cardiac medicines need to be achieved post-ACS to derive evidencebased benefits, including reductions in mortality, hospital readmission and improved symptom control. High intensity statin therapy can be started immediately at high-target dose, whilst ACE inhibitors (ACEi) and beta-blockers require up-titration to reach maximum tolerated doses. Despite a high percentage of patients being discharged on appropriate secondary prevention post-ACS, doses of ACEi and beta-blockers at the point of discharge are not at target dose due to haemodynamic effects and require up-titration post discharge. The aim of this study is to assess doses of ACEi and beta-blockers at the point of discharge and at various time points following discharge to assess rates of up-titration.

Method Patients discharged following ACS in September 2015, April 2016, July 2016 and September 2016 were identified using the Myocardial Ischemia National Audit Project (MINAP). Using electronic patient records, discharge doses of ACEi (or ARBs), beta blockers and statins were recorded. Patients were contacted in October 2016 to assess current doses of ACEi (or ARBs), beta blockers and statins corresponding to rates of up-titration at 1, 3, 6 and 12 months post-ACS. Full dose equivalents (FDE) were used to standardise variability of dosing between different ACEi (or ARBs), beta blockers and statins. FDE ranged from 0 , those not on the class of medication, to 1 , those on maximum doses. Patients without a true diagnosis of ACS or those deceased were excluded from the study.

Results 635 patients were identified from MINAP, of which 349 met the inclusion criteria for analysis. Baseline characteristics were similar between groups. Up-titration occurred in 53 patients (15\%) taking beta blockers and 79 patients (23\%) taking ACEi (or ARBs); however optimal doses of beta blockers and ACEi (or ARBs) were only achieved in 32 patients (9\%) and 40 patients (11\%) respectively. Average FDE of beta blockers and ACEi (or ARBs) was 0.38 and 0.37 respectively. 270 patients $(77 \%)$ were taking optimal doses of statins, with an average FDE of 0.86 . No differences were seen with regards to up-titration of secondary prevention at different time points.

Conclusions Results demonstrate an opportunity to titrate doses of beta blockers and ACEi (or ARBs) to optimal doses following ACS. NICE suggests up-titration to maximum doses within 4-6 weeks of discharge; however, whilst there is a positive trend in the number of patients on optimum doses with time, up-titration is minimal with patients achieving one third of target doses up to one year post-ACS. Data from this study shows a growing need to support patients in the community to optimise cardiac medications for secondary prevention after an ACS.

\section{OFFSET OF TICAGRELOR PRIOR TO CORONARY ARTERY BYPASS GRAFT SURGERY (CABG) SURGERY}

Kok Weng Ow*, Mark Thomas, William Parker, Heather Judge, Robert Storey. The University of Sheffield

\subsection{6/heartinl-2017-311726.71}

Introduction Ticagrelor is a more potent platelet inhibitor than clopidogrel but also has a more rapid offset of inhibitory effect. The optimal timing of discontinuation of ticagrelor prior to coronary artery bypass graft (CABG) surgery is unknown. In the ONSET/OFFSET study of patients with stable coronary artery disease, ticagrelor's effects dissipated within 48-120 hours of discontinuation. However, there is evidence that the pharmacodynamics of antiplatelet therapy are significantly altered during an acute coronary syndrome (ACS) and the ONSET/OFFSET study results should not be extrapolated to ACS patients. Although the PLATO study suggested that it was safe to discontinue ticagrelor 48 hours prior to $\mathrm{CABG}$, regulatory authorities recommended that ticagrelor should be discontinued 5 days prior to CABG. The PLATO study also showed that ticagrelor was associated with fewer deaths following pulmonary adverse events and sepsis compared to clopidogrel. It is unknown whether ticagrelor's additional property of inhibiting cellular adenosine uptake might underlie this observation. The aim of this study was to characterise the offset of ticagrelor's activity in inhibiting platelets and also in inhibiting cellular adenosine uptake in ACS patients.

Methods Patients admitted with ACS, treated with ticagrelor and referred for CABG were recruited. Venous blood was drawn from patients at the following 6 timepoints: 2 hours (h), 24 hour, 48 hour, 72 hour, 96 hour, and 120 hour after the last dose of ticagrelor prior to CABG. Whole-blood aggregometry was carried out using the Multiplate analyzer with adenosine diphosphate $6.45 \mu \mathrm{M}$ as agonist and the final value of area under the curve (AUC) (units, U) was recorded. Results were analysed using linear mixed models. A local protocol was referred to, where an AUC of $>50 \mathrm{U}$ supports safe progression to surgery, while values $50 \mathrm{U}$ indicates need for retesting 24-48 hours later. Inhibition of adenosine uptake was determined by measuring the adenosine plasma concentration using liquid chromatography.

Results 13 patients were recruited, all of whom received ticagrelor prior to CABG surgery. At 96 hour post ticagrelor, the mean AUC was $82.8 \mathrm{U}$, (95\% confidence interval, 60.4, 105.2). Only at 96 hour was the lower limit of the confidence interval $>50$ U. From the mixed model analysis, the comparison between 72 hour and 120 hour post ticagrelor showed a significant difference $(p=0.007)$ while 96 hour and 120 hour showed no significant difference $(\mathrm{p}=1.000)$. Adenosine plasma concentration was measured in 7 patients who received ticagrelor and showed no significant difference in adenosine plasma concentration across all timepoints.

Conclusion ACS patients might be safe to undergo CABG surgery 4 days after the cessation of ticagrelor. Ticagrelor might have no measurable effect on adenosine transport in ACS patients. 
Multiplate Area Under Curve ADP

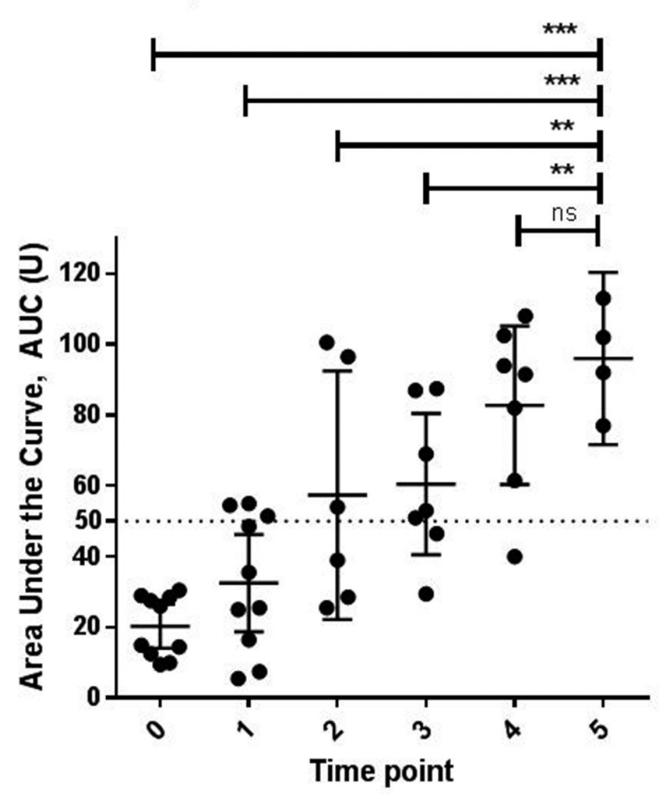

Abstract 72 Figure 1

\section{HPLC Measurements}

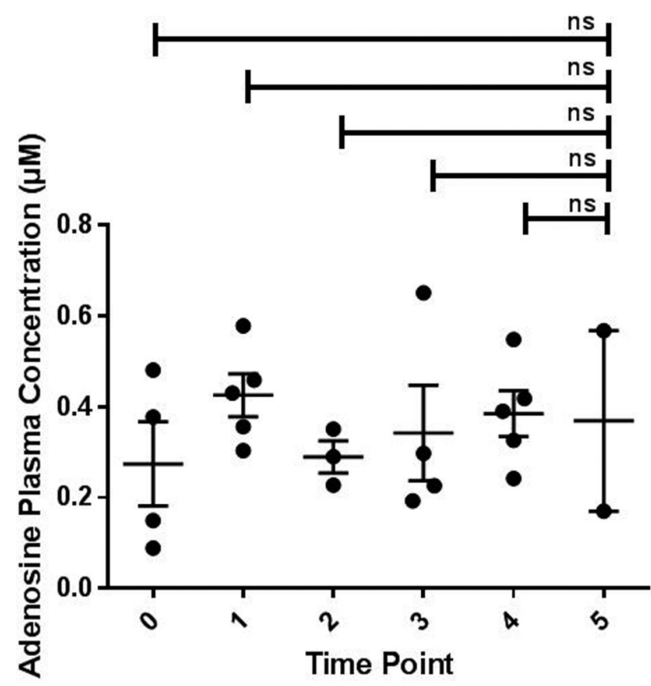

Abstract 72 Figure 2

\section{Congenital Heart Disease}

\section{IMPORTANCE OF CLASSIFICATION AND REASSESSMENT IN PATIENTS WITH PAH-CHD: THE NORPAP DATABASE}

${ }^{1}$ Dilip Abraham, ${ }^{2}$ Ruth Bingham*, ${ }^{3}$ Ranu Ranu, ${ }^{1}$ Catherine Head, ${ }^{4}$ Clive Lewis, 'Leisa J Freeman. ${ }^{1}$ Norfolk and Norwich University Hospitals NHS Foundation Trust; ${ }^{2}$ University of East Anglia; ${ }^{3}$ Baral; ${ }^{4}$ Papworth Hospital NHS Foundation Trust

\subsection{6/heartjnl-2017-311726.72}

Background Patients with Pulmonary Arterial Hypertension associated with Congenital Heart Disease (PAH-CHD) are subdivided into 4 categories: 1) Eisenmenger Syndrome, 2) $\mathrm{PAH}$

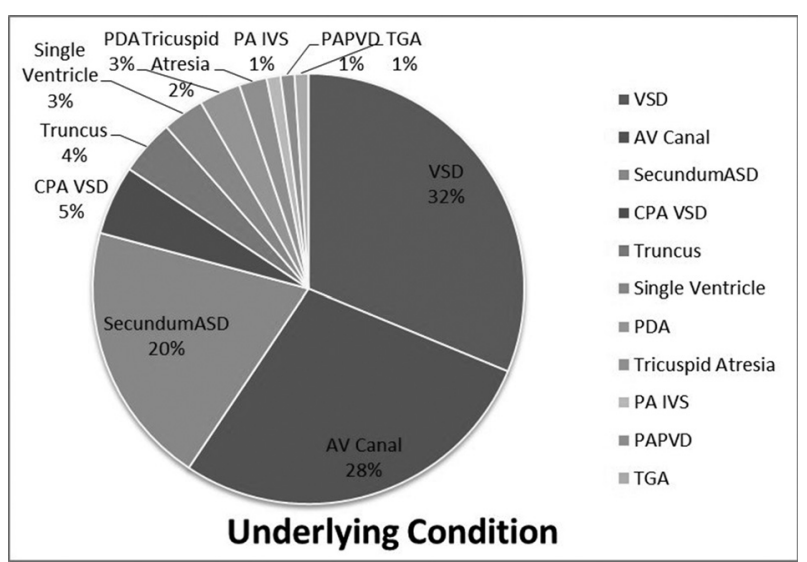

Abstract 73 Figure 1

with systemic-to-pulmonary shunts, 3) PAH with coincidental defects, 4) PAH after defect correction. There is clear benefit for targeted therapy in Category 1, 2 (less so in those with segmental PAH), and 4.

Methodology The NORPAP database was created in 1993 and contains 2854 patients. Patients in Norwich and Papworth have access to the joint GUCH-PVDU (Grown Up Congenital Heart Disease - Pulmonary Vascular Disease Unit) service. Patients with PAH-CHD were extracted from the NORPAP database and the above classification system applied.

Results 98/2854 (3.4\%) of patients had PAH-CHD. Female: male ratio 1.65:1. Age range 18-96, mean age 47, median 43.5. Trisomy 21 in 40 patients. Underlying condition shown in Figure 1. Number of patients per Category is as follows (Figure 2): Category $1 \quad(71 / 98=72 \%$, Age Range $=25-92$, median 42), Category $2(18 / 98=18 \%$, Range 18-96, median 58), Category 3 (3/98=3\%, Range 38-90, Median 42), Category $4 \quad(6 / 98=6 \%$, Range 24-69 Median 45: diagnoses DORV, VSD, TGA (atrial switch), PDA, PA-VSD, secundum ASD). 2 patients in category 4 had mixed aetiology with Chronic Thromboembolic Pulmonary Hypertension (CTEPH).

26/98 (27\%) of patients have died. Median age at death 41. Category 1: 21/71 29.5\%, Category 2: $2 / 18=11 \%$, Category $3: 1 / 3=33 \%$, Category $4: 2 / 6=33 \%$.

$55 / 98(56 \%)$ are on targeted therapy. In the Eisenmenger group $41 / 71(58 \%)$ are on targeted therapy. Sildenafil 11/ $41=26.8 \%$, Bosentan 13/41=31.7\%, Ambrisentan 1/41=2.4\%, two agent therapy $16 / 41=39 \%$.

Pre-treatment $6 \mathrm{~min}$ walk mean $288.5 \mathrm{~m}$, post-treatment mean $320.6 \mathrm{~m}$. Paired two sample t-test analysis reveals a significant difference in 6 min walk test (t-stat 2.02, p 0.01).

Of note was 28 year old Patient X: Trisomy 21 with large VSD and subpulmonary stenosis and suggestion of double outlet RV on poor quality echocardiography. Reassessed with cardiac MRI which confirmed tetralogy of Fallot. Following RHC, which showed RVOT gradient of $18 \mathrm{mmHg}$, mean PA pressure 55, PVR 25 wood units falling to 15.8 with maximal pulmonary vasodilatation, he was started on sildenafil.

Conclusion Targeted therapy in PAH-CHD improves quality of life, delays time to deterioration and may have a mortality benefit.Our limited data supports the evidence that targeted therapy improves objective exercise tolerance. Adult follow up surveillance clinics must be alert to deterioration of functional class in Category 1; Trisomy 21 patients that have been labelled as $\mathrm{PAH}$, but may have normal PA pressure at right 